\title{
Que Vidas Salvar? Escassez de Leitos de UTI, Critérios Objetivos de Triagem e a Pandemia da COVID-19
}

\author{
George Marmelstein
}

Faculdade 7 de Setembro, Fortaleza/Ceará, Brasil. E-mail: georgemlima@yahoo.com.br

Ana Carolina Morozowski

Juíza Federal da Justiça Federal do Paraná, Curitiba/Paraná, Brasil.

\section{Resumo}

Durante a pandemia da COVID-19, muitos países precisaram lidar com o risco do colapso do sistema de saúde, provocado pelo aumento da demanda por leitos de UTI e equipamentos de ventilação mecânica. Em alguns casos, foi necessário realizar escolhas trágicas para definir quem teria prioridade em receber o tratamento intensivo. Vários órgãos de saúde no mundo todo elaboraram guidelines com parâmetros racionais, transparentes e objetivos para orientar a tomada de decisão. O presente estudo é um esforço de sistematização do que foi produzido durante a fase mais crítica da pandemia, a fim de compreender os principais critérios que orientaram a ação dos órgãos reguladores. Palavras-chave

Direito à saúde; Escassez; UTI; Colisão de Direitos; COVID-19.

\section{Which Lives to Save? Shortage of ICU Beds, Objective Screening Criteria and the COVID-19 Pandemic}

\begin{abstract}
During the COVID-19 pandemic, many countries had to deal with the risk of the health system collapsing, due to the increased demand for ICU beds and mechanical ventilation equipment. In some cases, tragic choices needed to be made to define who would have priority in receiving critical treatment. Several health agencies around the world developed guidelines with rational, transparent and objective parameters to guide decision-making. The present study is an effort to systematize the main guidelines that arose at the critical stage of the pandemic, in order to understand the criteria that oriented the regulatory bodies and provide a guide to build a model adequate to the brazilian reality, considering a legal perspective.
\end{abstract}

Keywords

Right to health; Scarcity; ICU; Collision of Rights; tragic choices; COVID-19. 


\section{Sumário}

Introdução; 1. Princípios Fundamentais da Triagem Baseados em Fatores Clínicos; 1.1. Princípio da maximização do bem-estar; 1.1.1. Salvando mais vidas; 1.1.1.1. Resolução CFM n. 2.156/2016; 1.1.2. Salvando mais vidas e mais anos de vida - Modelo de Pittsburgh; 1.1.2.1. Escore de falência de órgãos (SOFA) para salvar mais vidas; 1.1.2.2. Análise das comorbidades para salvar mais anos de vida; 1.1.3. Salvando mais anos de vida ajustados pela qualidade; 1.1.3.1. QALY; 1.1.3.2. Escala Clínica de Fragilidade (CFS); 1.1.3.3. Diretriz do NICE - The National Institute for Health Care Excellence; 1.1.3.4. Protocolo AMIB de alocação de recursos durante a pandemia; 2. Princípios Fundamentais da Triagem Baseados em Fatores Não-Clínicos; 2.1. Princípio da igualdade e proibição de discriminação; 2.1.1. O critério etário como fator de desempate; 2.1.2. Escolha randômica como fator de desempate; 2.1.3. A triagem cega (blinded triagem); 2.2. Autonomia do paciente; Conclusões e Recomendações; Referências.

\section{Introdução}

A crise global provocada pela COVID-19 tem demonstrado que, mesmo em países desenvolvidos, a estrutura hospitalar existente pode não ser capaz de atender a todos os que necessitem de tratamento médico. Em muitos lugares, o número de pacientes contaminados que precisaram e precisam de unidades de terapia intensiva (UTI) e aparelhos de ventilação mecânica tendeu a exceder o número de equipamentos disponíveis e de profissionais especializados.

Nesse cenário, muitas pessoas que teriam que ser tratadas com a devida internação e eventual utilização de aparelhos de ventilação mecânica podem falecer pela incapacidade do sistema de oferecer tratamento adequado. É uma dramática situação de escassez, em que a demanda de pacientes supera a oferta de leitos de UTI disponíveis, acarretando mortes que seriam evitáveis em um momento de normalidade.

Decidir quem ocupará leitos de UTI em contexto de escassez é necessariamente uma escolha trágica, pois aqueles que forem excluídos do tratamento terão muito menos chances de sobreviver. Uma decisão tão impactante precisa ser tomada com base em critérios racionais, transparentes e objetivos, observando algumas diretrizes éticas que possam dar mais legitimidade, segurança e previsibilidade às escolhas tomadas pelos órgãos decisores.

Em várias partes do mundo, foram desenvolvidos guidelines para ajudar os profissionais responsáveis pela triagem a terem parâmetros justos de priorização de leitos em situação de escassez, sobretudo em face da pandemia da COVID-19. 
O debate não preocupou apenas países em que o sistema de saúde é precário e subfinanciado, mas até mesmo países bem desenvolvidos que possuem robustos sistemas de saúde, como o Reino Unido (NICE, 2020), Canadá (GOVERNMENT OF CANADA, 2020), Israel (SOLNICA E OUTROS, 2020), Itália (VERGANO E OUTROS, 2020), Suíça (SWISS, 2020), França (ARIE, 2020), Estados Unidos (WHITE, 2020) e vários outros.

No presente artigo, serão sistematizadas algumas orientações obtidas a partir desses estudos, a fim de ajudar a compreender quais são os fatores mais relevantes a serem ponderados para auxiliar na construção de um modelo decisório que seja adequado não apenas sob a perspectiva do direito à saúde, mas também dos demais direitos fundamentais envolvidos.

A ideia não é oferecer respostas prontas, mas apenas iniciar um debate que é complexo, dinâmico e sensível, já que tem o potencial de afetar a vida de milhões de seres humanos. Afinal, o que está em jogo, é uma pergunta bastante inquietante: diante de uma situação trágica, que vidas devem ser salvas?

\section{Princípios Fundamentais da Triagem Baseados em Fatores}

\section{Clínicos}

Dois pacientes em estado grave chegam ao mesmo tempo a um hospital público. Ambos precisam urgentemente de um leito de UTI. O problema é que só há uma vaga disponível naquele momento. Qual paciente deve ocupar a referida vaga?

Não há, e provavelmente nunca haverá, um consenso universal sobre quais critérios devem ser aplicados. Existem, na verdade, diversos manuais de orientações (guidelines), adotados por unidades de saúde no mundo todo, com uma variedade muito ampla de parâmetros que devem pautar a decisão médica.

Em uma perspectiva de senso comum, parece haver um apelo intuitivo para destinar a vaga para aquele paciente que se encontra no estado clínico mais grave. Afinal, este é o paciente que morrerá mais rápido caso nada seja feito.

Há, contudo, um sério problema de compreensão sobre a função da UTI nessa forma de pensar. Quando se fala em medicina de cuidados intensivos ou de cuidados críticos, a gravidade da condição clínica do paciente é pressuposta. Ou seja, os que estão concorrendo a um leito de UTI são os pacientes com risco de falecer. Portanto, mesmo um paciente em um estado clínico menos grave poderá ter a sua situação agravada e vir a óbito caso não seja internado imediatamente. 
Além disso, em algumas situações, o paciente que apresenta uma condição clínica mais grave pode ser aquele que tem menos chance de sobrevivência. Nesse caso, se o único leito disponível for destinado ao paciente com menos chance de recuperação, é possível que nenhum dos dois pacientes sobreviva, ocasião em que os recursos médicos não estariam produzindo qualquer benefício em termos de proteção da saúde.

Conforme se verá, a gravidade da condição clínica é um critério a ser levado em conta na admissão, mas em uma direção oposta àquela pressuposta pelo senso comum. Quanto mais grave for o estado de saúde do paciente, menor será a probabilidade de sobrevivência, e isso pode diminuir as suas chances de ser admitido no tratamento intensivo. Isso porque, em uma situação de escassez, devem ser favorecidos, dentre os pacientes que estão em estado crítico, aqueles que, de fato, necessitem da intervenção de suporte à vida e, ao mesmo tempo, possuam maior probabilidade de recuperação. A razão disso ficará clara mais à frente.

Outro critério que pode ser adotado para alocação de recursos escassos é o critério cronológico ou temporal conhecido como "primeiro a chegar, primeiro a ser servido" ("first come, first served"). Nesse modelo, cria-se uma fila de espera baseada na ordem cronológica de chegada até que todos os pacientes que necessitam de intervenções de suporte à vida possam ser internados.

É um critério que tem algumas vantagens por ser objetivo, por ser prático e por favorecer aqueles estão esperando há mais tempo. Apesar disso, ele também traz algumas objeções.

Em primeiro lugar, não é um critério eficiente, podendo agravar ainda mais o problema da escassez, pois não proporciona um ótimo aproveitamento dos recursos disponíveis. Na prática, alguns leitos seriam alocados para pessoas com poucas chances de sobrevivência, apenas por terem chegado primeiramente, impedindo que pacientes com alta probabilidade de recuperação sejam tratados. Além disso, pode beneficiar pessoas com pouca expectativa de vida, sem proporcionar a maximização global dos "anos de vida", que, como veremos, é outro critério de uso racional dos recursos médicos escassos.

Em segundo lugar, a priorização dos que chegam primeiro traz alguns problemas de equidade, na medida em favorece aqueles que estão mais próximos dos centros médicos e que possuem maior capacidade de mobilidade para chegar aos locais de atendimento. Assim, é possível que uma pessoa que necessite do tratamento há mais tempo seja preterida apenas porque não conseguiu chegar rapidamente ao hospital ou porque não teve um diagnóstico tão célere por falta de acesso aos serviços de saúde.

Outro problema do princípio "primeiro a chegar, primeiro a ser servido" envolve as políticas públicas de combate às pandemias. É provável que muitas pessoas que foram contaminadas nos momentos iniciais da pandemia sejam as que não seguiram as recomendações 
de proteção, inclusive o distanciamento social. Por outro lado, as que foram mais cuidadosas e só foram contaminadas mais tarde encontrarão um sistema de saúde colapsado, sendo preteridas pelas que chegaram antes. Em tese, o princípio temporal estaria criando um estímulo não desejado para que algumas pessoas sejam menos cuidadosas na fase inicial da pandemia (DUBNER, 2020).

Por conta dessas objeções ao "first come, first served", vários estudos condenam esse critério, sobretudo em tempos de pandemia, apresentando outras soluções mais eficientes e mais justas visando a maximização do bem-estar (EMANUEL E OUTROS, 2020).

\subsection{Princípio da maximização do bem-estar}

Os dois critérios acima citados (priorizar quem está em estado mais grave e priorizar quem chega primeiro) costumam ser rejeitados nas diretrizes de triagem porque focam essencialmente na posição individual de cada paciente sem levar em conta uma perspectiva mais ampla de usar os recursos escassos com racionalidade em benefício da coletividade.

Quando lidamos com a saúde pública, não estamos preocupados apenas com a saúde de um indivíduo, mas com a saúde de uma larga parcela da população (WHITE \& LO, 2020). Para que um sistema público considerado eficiente, ele precisa ser capaz de proporcionar a maior quantidade de benefícios para o maior número de pessoas.

Por isso, no coração dos principais guidelines elaborados pelas instituições de saúde mais avançadas do mundo, há uma preocupação com o princípio da maximização do bem-estar geral. A ideia é que os recursos escassos devem ser alocados de modo a favorecer o maior número de pessoas em termos quantitativos e qualitativos.

O princípio da maximização do bem-estar tende a adotar três pilares fundamentais que podem se interconectar em uma mesma dinâmica de triagem: (a) priorizar as escolhas que salvem o máximo de vidas possível (saving lives); (b) priorizar as escolhas que salvem o máximo de anos de vida possível (saving life years); (c) priorizar escolhas que salvem o máximo de anos de vida ajustados com a qualidade (saving QALY - Quality-adjusted life year).

Antes de explicar o significado prático de cada um desses parâmetros, é preciso esclarecer que não há unanimidade na forma de integrar essa multiplicidade de fatores potencialmente conflitantes. Em geral, os guidelines estabelecem pontuações, construídas a partir da conjugação desses critérios, que produzem uma hierarquia de prioridade que pode servir de base para decidir quem receberá o tratamento intensivo e quem receberá tratamentos alternativos (como o cuidado paliativo, o cuidado semi-intensivo ou o tratamento ambulatorial). 


\subsubsection{Salvando mais vidas}

O primeiro parâmetro é alicerçado na chance de recuperação, priorizando o tratamento daqueles pacientes em estado crítico que podem vir a sobreviver com o cuidado intensivo. Essa é a essência da preocupação em maximizar a quantidade de vidas salvas (saving lives), que é o parâmetro-base para a admissão de pacientes em leitos de UTI em várias partes do mundo, inclusive no Brasil.

\subsubsection{Resolução CFM n. 2.156/2016}

O modelo brasileiro, previsto na Resolução do Conselho Federal de Medicina n. 2156/2016, adota, em certo sentido, um sistema baseado na maximização de vidas salvas, criando uma hierarquia de cinco níveis de prioridade de modo a favorecer os pacientes com alta probabilidade de recuperação ${ }^{1}$.

Nesse modelo, há uma clara tentativa de otimizar a alocação de leitos de UTI, atribuindo um maior grau de prioridade para aqueles que precisam da intervenção de suporte à vida e, ao mesmo tempo, possuem mais chances de recuperação, prevendo tratamentos alternativos (nãointensivos) para os pacientes de prioridade mais baixa².

Apesar de ser um inegável avanço diante de um cenário anterior em que não havia qualquer critério para orientar as decisões médicas, a Resolução CFM n. 2156/2016 possui três problemas básicos: (a) primeiro, ela não resolve completamente o problema da alocação de leitos escassos, na medida em que não estabelece critérios de desempate entre pessoas com o mesmo nível de prioridade. Assim, ainda há uma lacuna caso exista apenas uma vaga de UTI e dois ou mais pacientes "com alta probabilidade de recuperação e sem nenhuma limitação de

${ }^{1} \mathrm{O}$ art. 60 da Resolução coloca no nível mais de prioridade aqueles pacientes "com alta probabilidade de recuperação e sem nenhuma limitação de suporte terapêutico" (Prioridade 1). No segundo nível, estão os "pacientes que necessitam de monitorização intensiva, pelo alto risco de precisarem de intervenção imediata, e sem nenhuma limitação de suporte terapêutico" (Prioridade 2). Em seguida, estão os "pacientes que necessitam de intervenções de suporte à vida, com baixa probabilidade de recuperação ou com limitação de intervenção terapêutica" (Prioridade 3 ) e os "pacientes que necessitem de monitorização intensiva, pelo alto risco de precisarem de intervenção imediata, mas com limitação de intervenção terapêutica" (Prioridade 4). Por sua vez, o nível mais baixo de prioridade envolve "os pacientes com doença em fase de terminalidade, ou moribundos, sem possibilidade de recuperação" (Prioridade 5). A nova Resolução CFM n. 2.271/2020, que define o que são as unidades de terapia intensiva e as unidades de cuidado intermediário, reafirma a necessidade de seguir tais critérios no item 3.1, do seu Anexo I.

2 Os arts. 70 e 8ㅇ da Resolução estabelecem que os pacientes que não são de Prioridade 1 e 3 devem ser prioritariamente admitidos em unidades de cuidados intermediários ou semi-intensivas (Prioridade 2 e 4) ou em unidades de cuidados paliativos (Prioridade 5, ou seja, pacientes em estágio mais grave, com doença em processo terminal irreversível e sem possibilidade de recuperação). A admissão desses pacientes em uma UTI é medida excepcional, a ser justificada de acordo com as "peculiaridades do caso e condicionado ao critério do médico intensivista” (art. 6o, da Resolução CFM n. 2156/2016). 
suporte terapêutico" necessitem de intervenção de suporte à vida ao mesmo tempo; (b) segundo, ela também não estabelece com clareza quais os critérios para definir se um paciente tem ou não "alta probabilidade de recuperação", dando margem para subjetividade, incerteza e arbítrio na escolha; (c) terceiro, ela foca apenas na perspectiva de "salvar vidas" (chance de recuperação), sem levar em conta os anos de vida, nem a qualidade da sobrevida, que também deveriam ser considerados em uma perspectiva de maximização do bem-estar.

\subsubsection{Salvando mais vidas e mais anos de vida - Modelo de Pittsburgh}

Um guideline mais avançado foi elaborado por professores da Faculdade de Medicina da Universidade de Pittsburgh, com o título "Allocation of Scarce Critical Care Resources During a Public Health Emergency" (WHITE, 2020). Esse guia tem sido adotado por algumas unidades de saúde nos Estados Unidos e prevê certos critérios fundamentais de alocação de recursos de cuidado crítico para orientar as decisões médicas, especialmente em períodos de emergência (WHITE, 2020).

Dentro dessa proposta, todos os pacientes que atenderem às indicações médicas habituais para leitos e serviços de UTI recebem uma pontuação de prioridade de 1 a 8 , sendo que quanto mais baixa for a pontuação maior é a prioridade.

Essa pontuação é estabelecida com base em uma probabilidade de os pacientes sobreviverem à alta hospitalar, avaliada objetivamente a partir de critérios clínicos previamente estabelecidos. Também leva em conta a presença de condições médicas subjacentes que limitam severamente o prognóstico para sobrevida no curto prazo, mesmo que o paciente consiga sobreviver após os cuidados críticos.

\subsubsection{Escore de falência de órgãos (SOFA) para salvar mais vidas}

Para calcular a probabilidade de sobrevivência, o guideline de Pittsburgh sugere o uso do escore SOFA - Sequential Organ Failure Assessment, que é um parâmetro de medida para verificar as condições de saúde de pacientes gravemente enfermos, inclusive suas chances de sobrevivência. De modo simplificado, a pontuação SOFA se baseia na probabilidade de determinados órgãos vitais falharem, a partir da análise de seis funções fisiológicas: respiratória, hepática, 
cardiovascular, renal, coagulatória e neurológica. Quanto maior for o nível de falha dos órgãos, maior é a pontuação SOFA. A prioridade é dada para aqueles que, estando em estado crítico, têm a pontuação SOFA mais baixa, pois isso representa uma maior chance de sobrevivência.

Uma das vantagens do uso do SOFA como parâmetro de priorização de pacientes é que é um escore relativamente prático de ser calculado e já é usado em várias unidades de tratamento intensivo no mundo todo, inclusive no $\mathrm{Brasil}^{3}$. O único problema desse critério é que ele só leva em conta a chance de recuperação, maximizando o princípio saving lives, sem levar em conta nem a quantidade, nem a qualidade de anos de vida que serão salvos.

\subsubsection{Análise das comorbidades para salvar mais anos de vida}

Por essa razão, o guideline da Faculdade de Pittsburgh sugere que, na escala de prioridades, também seja incluído um critério visando maximizar os anos de vida (saving years of life). 0 objetivo é levar em conta o tempo de sobrevivência após a alta hospitalar, visando priorizar aqueles que poderão viver mais tempo.

Para entender por que isso é relevante, tome-se uma situação hipotética em que há dois pacientes aguardando um leito de UTI, sendo que ambos possuem uma condição clínica semelhante, de acordo com o escore SOFA. Ou seja, a probabilidade de sobrevivência após os cuidados intensivos é semelhante para os dois pacientes. Ocorre que um dos pacientes não tem qualquer problema de saúde além daquele que o levou ao estado crítico, enquanto o outro é portador de um câncer em estágio avançado e, portanto, sua expectativa de vida é mais curta. Nesse caso, o princípio da maximização dos anos de vida sugere que a prioridade deve ser dada ao paciente que não possui outras comorbidades, pois o cuidado crítico para o paciente com câncer gerará apenas um benefício de curto prazo.

Nesse contexto, o guideline de Pittsburgh sugere que a presença de condições médicas em um estado avançado deveria ser usada para diminuir o nível de prioridade daqueles pacientes cujo prognóstico indicam que possuem pouca expectativa de vida. O guia não lista quais são as condições clínicas que devem ser levadas em conta para reduzir o grau de prioridade, mas indica que são aquelas enfermidades que estejam associadas à morte em um prazo de um a cinco anos, avaliadas individualmente pelo médico, com base nas melhores evidências médicas objetivas

\footnotetext{
${ }^{3}$ O Comitê de Ética do Hospital de Clínicas da Unicamp sugere que, no Brasil, seja associado o SOFA com as indicações de terapia intensiva do CFM para decidir se a pessoa deve ou não ir para a UTI, justificando essa proposta com o fato de que o escore SOFA é usado em terapia intensiva há muito tempo e é bem consistente (MANIR, 2020).
} 
disponíveis (WHITE, 2020). Assim, na prática, se o paciente tiver alguma doença que reduza significativamente a sua expectativa de vida, com probabilidade de vir a óbito nos próximos anos em razão da doença, esse dado deve ser contabilizado no seu escore de prioridade juntamente com o seu escore SOFA.

\subsubsection{Salvando mais anos de vida ajustados pela qualidade}

Outro conceito que costuma estar associado à maximização do bem-estar é conhecido como QALY - Quality Adjusted Life Years, comumente traduzido como anos de vida ajustados pela qualidade.

\subsubsection{QALY}

O QALY é uma medida que combina a quantidade de sobrevida (duração) com a sua respectiva qualidade, gerando um número que funcionará de base de comparação para orientar as decisões em medicina. Para determinar o QALY, multiplica-se o valor da utilidade associado a um determinado estado de saúde pelos anos vividos nesse estado. Um ano de vida vivido em um estado perfeitamente saudável vale 1 QALY (ou seja, 1 ano de vida x 1 valor unitário de qualidade). Um ano de vida vivido em um estado de saúde inferior à perfeita, vale menos de 1 QALY. Por exemplo, 1 ano de vida vivido em uma situação com utilidade/qualidade 0,5 recebe 0,5 QALY (WEINSTEIN E OUTROS, 2009) ${ }^{4}$.

Embora o QALY seja um critério bastante utilizado em decisões envolvendo a alocação de recursos de saúde, como por exemplo para estimar o custo-efetividade de uma determinada tecnologia médica, ou para orientar a ordem de prioridade nas filas de transplante ou de cirurgias eletivas, o seu uso na medicina intensiva é menos aceito.

As objeções envolvendo a aplicação do QALY na definição de prioridades em cuidados críticos envolvem: (a) primeiro, uma dificuldade de operacionalização, já que o seu cálculo exige a avaliação complexa de uma grande quantidade de informações que nem sempre estão disponíveis no contexto de uma UTI, onde as respostas devem ser urgentes, e o histórico clínico

${ }^{4} \mathrm{O}$ valor de utilidade/qualidade é estabelecido por meio de alguns métodos desenvolvidos pela economia, como o time-trade-off (TTO), standard gamble (SG), visual analogue scale (VAS) e o questionário EQ-5D, do EuroQol Group (MURPHY, 2018). Para os fins do presente estudo, não é necessário aprofundar os detalhes de cada um desses métodos. Basta dizer que eles se baseiam na ponderação de escolhas subjetivas realizadas por uma determinada quantidade de pessoas, que indicam se preferem um determinado estado de saúde a outro, ou o quanto estariam dispostos a arriscar a sua vida na realização de uma determinada intervenção cirúrgica. Ao fim e ao cabo, tem-se um número que representa o resultado de uma comparação entre o estado perfeito de saúde e o nível de desconforto gerado por um tipo específico de enfermidade. 
do paciente nem sempre é completo; (b) segundo, os critérios para estabelecer o valor da utilidade-qualidade não são universalmente aceitos, gerando muitas críticas quanto à sua objetividade e confiabilidade, pois se baseiam em generalizações de preferências subjetivas estabelecidas em cenários meramente hipotéticos e não reais; (c) por fim, o QALY pode gerar um problema de equidade, pois a análise da qualidade da sobrevida tende a ser prejudicial às pessoas com deficiência e aos idosos que, em geral, estão sujeitos a uma condição de saúde menos perfeita.

Em razão disso, são raros os guidelines que preveem, de modo explícito e direto, a utilização do QALY como parâmetro decisório na distribuição de leitos, optando por parâmetros alternativos como a Escala Clínica de Fragilidade (CFS).

\subsubsection{Escala Clínica de Fragilidade (CFS)}

A Escala Clínica de Fragilidade - Clinical Frailty Scale - CFS (também conhecida como The Rockwood Scale), é uma tabela prática, de uma página apenas, que enumera 9 níveis de fragilidade, começando de muito saudável (very fit) até chegar aos doentes terminais (terminally ill) $)^{5}$.

Embora a CFS tenha sido desenvolvida primariamente para servir como uma ferramenta prática de avaliação multidimensional do estado físico das pessoas idosas, o seu uso clínico tem sido ampliado para auxiliar julgamentos em várias outras áreas, inclusive como parte de uma avaliação médica que possa servir de suporte às decisões de admissão ou não de pacientes em leitos de UTI (CHOL, 2016).

\footnotetext{
${ }^{5}$ Eis todos os níveis de fragilidade previstos na CFS: (1) very fit ou muito saudável: pessoa robusta, com energia e motivação. Realiza exercícios físicos regulares e está entre os melhores para a sua idade; (2) well ou bem de saúde: pessoa sem doença ativa, realiza exercícios físicos regularmente, mas é menos hígida do que as da categoria anterior; (3) managing well ou com doenças sob controle: pessoa com sintoma de doenças, mas controladas, que não é regularmente ativa; (4) vulnerable ou vulnerável: apesar de não ser dependente, muitas vezes a sua condição de saúde leva à redução de atividades; (5) mildly frail ou ligeiramente frágil: com dependência limitada para atividades instrumentais da vida privada (AIVD) de alta ordem (finanças, trabalhos domésticos, medicamentos); (6) moderately frail ou moderadamente frágil pessoa que necessita de ajuda para atividades da vida diária (AVD) e AIVD. Em casa, apresenta problemas com escadas e precisa de ajuda com o banho e ajuda mínima para se vestir; (7) severely frail ou severamente frágil - completamente dependente para AVDs, estável e não possui risco expressivo de entrar em óbito (num período de 6 meses); (8) very severely frail ou muito severamente frágil - pessoa completamente dependente, aproximando-se do fim da vida; (9) terminally ill ou doente terminal aproxima-se da terminalidade. Esta categoria se aplica à pessoa com uma expectativa de vida menor do que seis meses e que é evidentemente frágil (CECHINEL, 2015).
}

Revista Publicum

Rio de Janeiro, v. 6, n. 1, p. 94-117, 2020

http://www.e-publicacoes.uerj.br/index.php/publicum

DOI: $10.12957 /$ publicum.2020.57573 


\subsubsection{Diretriz do NICE - The National Institute for Health Care}

\section{Excellence}

Um dos primeiros órgãos a adotar a CFS, como parâmetro de admissibilidade em UTI, foi o NICE The National Institute for Health Care Excellence, principal órgão de saúde pública da Inglaterra, no contexto específico da pandemia do novo coronavírus.

Em março de 2020, o NICE elaborou um guideline com o título "CODIV-19 rapid guideline: critical care in adults", para servir de orientação às decisões médicas envolvendo a admissão de adultos em leitos de UTI durante a pandemia, sugerindo explicitamente a aplicação da Escala Clínica de Fragilidade (CFS) (NICE, 2020).

No primeiro modelo proposto pelo NICE, qualquer adulto que tivesse uma condição de fragilidade acima de 5 (ou seja, que fosse ligeiramente frágil, com dependência limitada para atividades instrumentais da vida privada de alta ordem) deveria passar por uma avaliação mais detalhada para saber se a sua condição seria ou não apropriada para ocupar um leito de UTI. Nesse cenário, a decisão poderia ser no sentido de não internar o paciente, mesmo que a sua situação clínica fosse crítica, baseada em uma análise de risco-benefício.

Esse modelo sofreu severas críticas por ser potencialmente prejudicial às pessoas com deficiência e aos idosos, na medida em que diminui as suas chances de serem admitidas em leitos de UTI. Por conta disso, em abril de 2020, o NICE atualizou o seu guideline para abrandar o uso da Escala Clínica de Fragilidade. No novo modelo, a Escala Clínica de Fragilidade somente deve ser usada em pacientes com mais de 65 anos, sem incapacidades estáveis de longo prazo (por exemplo, paralisia cerebral), e ainda assim dentro de uma avaliação holística que leve em conta outros fatores, como a existência de comorbidades e as condições de saúde subjacentes. Nesse novo modelo, a CFS seria apenas um dos elementos dentro de uma análise de risco-benefício mais ampla a orientar o médico em sua decisão sobre internar ou não o paciente.

\subsubsection{Protocolo AMIB de alocação de recursos durante a pandemia}

A Associação de Medicina Intensiva Brasileira (AMIB) publicou, no dia 24/04/2020, o Protocolo AMIB de alocação de recursos em esgotamento durante a pandemia por COVID-19 (KRETZER E OUTROS, 2020). O instrumento representou um grande avanço em relação à Resolução CFM $n$. 2.156/2016, uma vez que trouxe detalhadamente critérios para decidir quem receberá tratamento intensivo em situação de extrema escassez. Uma semana depois, em 01/05/2020, o 
protocolo foi substituído por uma recomendação da AMIB de alocação de recursos em esgotamento durante a pandemia, da qual a ABRAMEDE (Associação Brasileira de Medicina de Emergência), a SBGG (Sociedade Brasileira de Geriatria e Gerontologia) e a ANCP (Academia Nacional de Cuidados Paliativos) também foram signatárias.

O primeiro protocolo trazia considerações importantes, tais como a necessidade de se dar publicidade aos processos de alocação de recursos, com o devido registro das razões das escolhas feitas, seguindo parâmetros objetivos. Reconhecia que as regras deviam seguir princípios éticos e legais, e não só médicos. Considerava essencial que cada hospital nomeasse membros para uma comissão de triagem (de preferência com 3 profissionais), alinhando o protocolo com o sistema de regulação de leitos "que permita encaminhamento de pacientes para outras unidades hospitalares com disponibilidade de leitos, incluindo a possibilidade de intercâmbio entre leitos públicos e privados". Também levava em conta o respeito à dignidade da pessoa humana, bem como à vontade do paciente. Recomendava que a triagem se aplicasse a todos os pacientes (COVID e não COVID), que os pacientes que não fossem contemplados com a vaga continuassem sendo tratados e que houvesse a possibilidade de revisão das escolhas feitas (o que exigiria que a fundamentação fosse clara e transparente). O protocolo trazia ainda a necessidade de que a triagem se estendesse para após a internação, com a análise da evolução dos pacientes internados, a fim de verificar se o prolongamento da vida era útil. Para tanto, o protocolo não estabelecia critérios, mas recomendava que “o tempo do 'trial terapêutico' deve ser proporcional ao tempo que normalmente leva para a recuperação de cada condição clínica específica".

Os fundamentos do protocolo eram: dar preferência a pacientes com melhores chances de benefício (salvar o maior número de vidas), com maiores expectativas de sobrevida (salvar o maior número de anos de vida), bem como equalizar a oportunidade de indivíduos de passar pelos diferentes ciclos de vida (equalizar oportunidades).

Com base nisso, adotou-se um modelo que levava em conta a pontuação SOFA, a existência de comorbidades com expectativa de sobrevida inferior a um ano e a identificação da faixa etária em que o paciente se encontrava. Com relação ao SOFA, o paciente ganharia 1 ponto se o seu SOFA fosse inferior ou igual a 8, 2 pontos se o SOFA ficasse entre 9-11, 3 pontos se ele ficasse entre 12-14, e 4 pontos se ele fosse superior a 14. Caso houvesse comorbidades que indicassem expectativa de sobrevida inferior a um ano, o paciente ganharia 3 pontos. Por fim, se o paciente tivesse entre 0-49 anos, ganharia 1 ponto, entre 50-69 ganharia 2 pontos, entre 70-84 ganharia 3 pontos e acima de 85, 4 pontos. Após essa análise, o paciente obteria uma pontuação e, quanto menor ela fosse, mais chances ele teria de conseguir um leito de UTI. Em caso de 
empate, deveriam ser utilizados os seguintes critérios de desempate, nessa ordem: 1) menor escore da Escala de Fragilidade; 2) menor escore SOFA total; e 3) randomização.

Note-se que o protocolo adotado impedia que pacientes fossem excluídos a priori. Além disso, adaptar-se-ia a diversos níveis de saturação do sistema de leitos e procurava evitar a discriminação etária como fator de desempate ou como fator por si só decisivo. Outro aspecto importante é que ele partia da premissa de que nunca haveria consenso em relação aos fatores considerados, tendo em vista a pluralidade de valores que uma sociedade engloba. Sobre isso, diz que "a exposição do protocolo ao debate público e a revisões pode contribuir com a busca de uma maior legitimidade ética do processo".

Tendo em vista algumas objeções, especialmente no que diz respeito à adoção do fator idade como um dos critérios determinantes para a triagem, um novo documento foi elaborado, conforme dito. O novo protocolo manteve as mesmas premissas éticas do anterior, bem como o propósito de salvar o maior número de vidas, salvar o maior número de anos/vida e de equalizar as oportunidades de passagem pelos diferentes ciclos de vida. Entretanto, houve a alteração dos critérios de triagem, a fim de evitar discriminações etárias.

Os dois primeiros critérios (SOFA total e comorbidades) continuaram sendo adotados. A mudança ocorreu no terceiro critério, que passou a considerar a funcionalidade pela escala desenvolvida pelo Eastern Cooperative Oncology Group (ECOG). A escala quantifica a capacidade funcional física e a capacidade de independência e de auto-cuidado do paciente. Quanto maior o escore obtido, que pode variar de 0 a 4 pontos, maiores são as chances de se obter um desfecho clínico desfavorável. Assim, se o paciente obtiver a pontuação 0 ou 1 na escala do ECOG, ele ganhará 1 ponto na triagem. Se ele obtiver 2 pontos na escala, ele ganhará 2 pontos na triagem e assim por diante.

Com base nos 3 critérios, calcula-se a pontuação total para que se verifique quem tem prioridade para a obtenção do leito. O paciente com a menor pontuação será o selecionado. Como fator de desempate, o protocolo sugere atribuir o leito ao que obtiver o menor score SOFA total e que for considerado prioritário pela equipe de triagem.

\section{Princípios Fundamentais da Triagem Baseados em Fatores}

\section{Não-Clínicos}

As considerações acima delineadas já nos permitem perceber que são várias as possibilidades de organizar uma ordem de prioridades para admissão em leitos de UTI, levando em conta alguns fatores clínicos que sejam relevantes para salvar mais vidas, salvar mais anos de vida ou salvar 
mais anos de vida com qualidade. Veremos agora alguns princípios de triagem baseados em fatores não-clínicos que também merecem ser considerados.

\subsection{Princípio da igualdade e proibição de discriminação}

O resultado de uma triagem médica pode significar a inclusão ou exclusão de determinadas pessoas em um atendimento médico adequado, necessário e urgente. Quando um paciente é considerado não elegível para ocupar um leito de UTI, isso não significa que ele não precisa dos cuidados críticos. Muitas vezes, a não admissão é fruto de um trade-off em que vários pacientes necessitados concorrem pela mesma vaga, e apenas um poderá ocupá-la.

Por conta disso, é imperioso que, na construção de parâmetros para a alocação justa de recursos escassos, seja adotado um princípio geral de igualdade, a fim de garantir que qualquer pessoa necessitada possa, em princípio, ter a chance de vir a usufruir o serviço médico. Todas as pessoas, nesse contexto, merecem ter iguais oportunidades de participar do processo de triagem, não podendo haver exclusões arbitrárias, preventivas e categóricas.

Por essa razão, merecem críticas os guidelines que excluem determinados grupos com base em critérios não necessariamente clínicos e até mesmo discriminatórios. Por exemplo, em alguns lugares, excluem-se pessoas com deficiência física ou mental, portadores do HIV, pessoas com baixo nível cognitivo ou baixo status socioeconômico e assim por diante, o que é claramente incompatível não só com o direito à saúde e à vida, mas também com o princípio de que todos devem ser tratados com igual respeito e consideração (BAKER \& FINK, 2020).

$\mathrm{Na}$ verdade, até mesmo fatores que podem ter relevância clínica, como doenças préexistentes, idade ou sexo, não deveriam funcionar como obstáculos absolutos de acesso a unidades de tratamento intensivo, podendo apenas ser considerados dentro de uma escala de pontuação que leve em conta o seu impacto no quadro clínico, especialmente na probabilidade de recuperação, na expectativa de vida e na qualidade de vida após o tratamento.

Em alguns guidelines, pacientes com problemas cardíacos ou com câncer, portadores de doenças degenerativas, respiratórias ou renais, por exemplo, são considerados, de plano, não elegíveis para ocupar um leito de UTI. Esses modelos que excluem preventivamente alguns grupos do processo de triagem também são bastante problemáticos do ponto de vista ético (e jurídico), pois tal exclusão implica uma negação absoluta do direito à saúde e do direito à vida, sem levar em conta outros fatores igualmente importantes que poderiam pesar a favor da pessoa excluída (WHITE \& LO, 2020).

Outro fator de exclusão muito polêmico é a idade. Na Itália, durante o colapso provocado pela COVID-19, no começo de 2020, a SIAARTI (Società Italiana di Anestesia Analgesia 
Rianimazione e Terapia Intensiva) orientou aos médicos que fosse utilizado um limite etário para admissão em unidades de tratamento intensivo. De acordo com a SIAARTI, "o princípio subjacente seria economizar recursos limitados, que podem se tornar extremamente escassos para aqueles que têm uma probabilidade muito maior de sobrevivência e expectativa de vida, a fim de maximizar os benefícios para o maior número de pessoas" (VERGANO E OUTROS, 2020). Segundo noticiado, em alguns hospitais, pacientes com mais de 80 anos foram excluídos da fila de espera de UTI com base nessa recomendação (MOUNK, 2020).

Em princípio, a exclusão de determinados pacientes em razão da idade avançada poderia ser justificada com base no princípio da maximização da quantidade de vidas salvas. Afinal, as pessoas mais idosas costumam estar em uma condição clínica que tende a diminuir as chances de sobrevivência. Porém, tal correlação entre idade avançada e má condição clínica não é sempre necessária. Em algumas situações, é possível que uma pessoa idosa possa ter mais chance de sobrevivência do que uma pessoa mais jovem, sendo injusto desconsiderar uma condição de saúde mais ampla apenas em razão da idade.

Por isso, é recomendável seguir a orientação de alguns modelos mais avançados que incorporam um princípio geral de igualdade de oportunidades, evitando estabelecer critérios discriminatórios apriorísticos e prestigiando a tomada de decisão com base em parâmetros clínicos objetivamente mensuráveis. A lógica dos modelos mais avançados é que nenhum grupo pode ser excluído de plano da oportunidade de ter acesso a um tratamento adequado. Em linha de princípio, todas as pessoas são consideradas elegíveis para participar de um processo de triagem e recebem uma pontuação obtida a partir de uma análise abrangente de tudo aquilo que pode ter relevância clínica.

O já mencionado "COVID 19 - DECISION SUPPORT TOOL", desenvolvido por médicos do NHS, apesar de seus problemas, ilustra essa preocupação. Em princípio, até mesmo pacientes com mais de 80 anos podem ser considerados elegíveis para ocupar um leito de UTI, desde que tenham uma boa condição física e não sejam portadores de comorbidades capazes de afetar a chance de sobrevivência. Do mesmo modo, pacientes que sejam portadores de comorbidades graves podem receber os cuidados críticos, caso o seu escore global não ultrapasse o total de 8 pontos.

É certo que a referida ferramenta utiliza explicitamente o critério etário como fator para aumentar ou diminuir a pontuação. Pessoas mais idosas já saem em desvantagem na contagem de pontos, e isso é, de fato, problemático do ponto de vista ético-jurídico. Como contraargumento, existe a justificativa de que, em se tratando da COVID-19, há uma forte correlação entre a idade e a taxa de letalidade (ou chance de sobrevivência), razão pela qual seria racional 
levar em conta o fator de idade no processo de triagem com base no objetivo de maximizar vidas salvas ${ }^{6}$.

Outro problema está relacionado com a Escala Clínica de Fragilidade (CFS), que também produz um impacto desproporcional nos grupos com idade mais avançada e nas pessoas com deficiência. O contra-argumento é que a própria ferramenta indica que o fator de fragilidade fisiológica só deve ser levado em conta quando houver impacto na probabilidade de recuperação, o que seria compatível com o objetivo de maximizar anos de vida. De qualquer modo, como as ressalvas ficam nas entrelinhas, há um risco de que a aplicação da CFS possa produzir um enviesamento contra pessoas idosas e com deficiência. O ideal seria criar salvaguardas mais eficientes para que a avaliação da idade e do grau de fragilidade não penalizem injustificadamente essas pessoas. Idade e comorbidades só devem ser levadas em conta se afetarem, comprovadamente, a probabilidade de recuperação e a quantidade de sobrevida. Do contrário, será um critério discriminatório ilegítimo.

\subsubsection{O critério etário como fator de desempate}

Em alguns guidelines, há uma defesa explícita de que a prioridade dos recursos escassos de leitos de UTI seja concedida às pessoas mais jovens, pelo menos como critério de desempate. $\mathrm{O}$ argumento envolve o princípio do ciclo de vida, que se baseia na premissa de que todo ser humano tem o direito de usufruir todos os estágios de uma vida plena. Assim, em uma situação em que todas as condições clínicas fossem idênticas, uma pessoa jovem teria prioridade de ser atendida por não ter vivido ainda todos os ciclos da vida (WHITE E OUTROS, 2020).

Apesar de ser um argumento razoável, a utilização da idade como fator de desempate é problemática por algumas razões.

Em primeiro lugar, as pessoas idosas estão protegidas pelo direito antidiscriminatório, somente sendo justificável criar tratamentos prejudiciais que tenham base racional e possam ser justificados por razões clínicas. O ciclo da vida, em princípio, não é uma razão clínica que possa

\footnotetext{
6 É preciso, contudo, ter bastante cautela na formulação dessas pontuações baseadas em correlações estatísticas, pois nem sempre correlação implica causalidade. Por exemplo, seria possível especular (para fins meramente argumentativos) que o COVID-19 gera uma taxa de letalidade maior nas pessoas idosas não por conta da idade em si, mas nas condições clínicas relacionadas à fragilidade e à existência de comorbidades. Nesse caso, o mais relevante do ponto de vista clínico não seria o fator etário, mas a existência de outras condições fisiológicas debilitantes. Esse cuidado deve ser ainda maior para não se criar uma "profecia autorrealizadora" que aumentaria ainda mais o prejuízo a grupos vulneráveis. Nesse cenário, um determinado grupo não receberia o tratamento adequado e, por isso, suas chances de sobrevivência seriam menores; depois, analisando a estatística, o mesmo grupo seria prejudicado na ordem de prioridade com base no argumento circular de que teria menos chance de sobrevivência.
}

Revista Publicum

Rio de Janeiro, v. 6, n. 1, p. 94-117, 2020

http://www.e-publicacoes.uerj.br/index.php/publicum

DOI: $10.12957 /$ publicum.2020.57573 
justificar um tratamento prejudicial, mesmo porque é possível que um paciente mais jovem tenha alguma condição de saúde (comorbidade) que reduza a sua expectativa de vida.

Em segundo lugar, nos modelos que utilizam parâmetros clínicos de triagem, a idade já é um fator que entra na equação. Em alguns casos, a idade é um fator diretamente considerado, inclusive para dificultar as oportunidades das pessoas mais idosas. Em outros casos, mesmo quando a idade não é diretamente mencionada, o uso de algumas escalas clínicas pode ter um impacto desproporcional indireto nas pessoas mais idosas, até porque há uma tendência natural de que a condição de saúde seja deteriorada com o passar dos anos. Assim, os idosos seriam prejudicados duas vezes: na fase de análise clínica e na fase de desempate.

Por fim, a ideia de ciclo de vida somente tem sentido quando se trata de pessoas com uma diferença substancial de idade. Seria injusto que uma pessoa de 22 anos tivesse prioridade sobre uma pessoa de 23 anos por causa de apenas um ano de diferença, sabendo que o benefício pode significar uma sobrevida de mais de 60 anos. Assim, seria preciso criar outro critério de desempate, a ser aplicado quando a diferença de idade não fosse significativa, o que tornaria o sistema desnecessariamente complexo.

Diante disso, o ideal é que o fator etário não seja levado em conta no processo de admissão em leitos de UTI, a não ser quando houver razões clínicas que possam justificar a decisão.

\subsubsection{Escolha randômica como fator de desempate}

Outro critério eticamente possível de desempate em cenários de incerteza, onde não existe base clínica objetivamente precisa para definir quem deve ter a prioridade de ocupar um leito de UTI, é a escolha randômica. A loteria da vaga tem o mérito de ser mais objetiva e potencialmente imune a vieses, além de proporcionar uma decisão simples e rápida, o que pode ser importante no contexto de uma unidade de tratamento intensivo. Por isso, esse costuma ser o critério de desempate adotado por alguns guidelines mais avançados, geralmente acompanhado de salvaguardas para garantir a transparência do processo de sorteio (EMANUEL, 2020).

O guideline proposto pela AMIB, por exemplo, sugere explicitamente que os critérios de desempate a serem adotados no Brasil levem em conta, sequencialmente, o escore de fragilidade clínica, a pontuação total SOFA e, por fim, a randomização (por sorteio). Quanto às salvaguardas, a AMIB sugere as seguintes: a) atendimento inicial pleno enquanto a triagem é realizada, idealmente dentro de um tempo de 90 minutos; b) a revisão regular dos critérios de triagem de cada paciente, incluindo a atualização das pontuações já que elas podem variar com a evolução do quadro; c) registro das pontuações de cada paciente triado em prontuário; d) revisão dos casos triados e não triados por uma equipe independente.

Revista Publicum

Rio de Janeiro, v. 6, n. 1, p. 94-117, 2020

http://www.e-publicacoes.uerj.br/index.php/publicum

DOI: $10.12957 /$ publicum.2020.57573 


\subsubsection{A triagem cega (blinded triage)}

Alguns guidelines, visando reduzir o enviesamento das escolhas por razões ilegítimas, sugerem que os órgãos de triagem tomem decisões com o ocultamento ou cegamento de algumas informações irrelevantes (blinded triage).

Esse procedimento funciona nas situações em que a decisão sobre a admissão ou não de pacientes em leitos de UTI é tomada por órgãos que não estão em contato direto com o paciente. O argumento é que a inclusão de dados como fotografia, identidade, profissão etc. poderia influenciar, de modo negativo, a decisão, na medida em que pode gerar preconceito implícito ou explícito.

Conforme explicado por Sabine Michalowski, o cegamento possivelmente reduziria o risco de subjetividade nas decisões de triagem, reduziria a carga psicológica na equipe clínica da linha de frente, daria maior eficiência, consistência e previsibilidade em relação à aplicação dos princípios de triagem, possibilitando ainda a preservação do médico como defensor do paciente. Há, contudo, algumas desvantagens, como uma possível perda de informações clínicas relevantes e o aumento da burocracia no processo decisório (MICHALOWSKI E OUTROS, 2020).

\subsection{Autonomia do paciente}

Há que se levar em conta, ainda, outro princípio de máxima importância no contexto da ética médica: a autonomia de vontade ou o consentimento livre e informado do paciente. Em muitos casos, é o próprio paciente que não deseja ser internado em unidades de tratamento intensivo, pois isso implica riscos, sofrimento e um prolongamento indesejado da existência. As chamadas diretivas antecipadas de vontade, que solicitam a limitação de suporte terapêutico, informando que não desejam manobras de ressuscitação (DNR - do not resuscitate) ou procedimentos de entubamento, hão de ser respeitadas, especialmente em tempos de pandemia, em que a morte em um hospital pode implicar ainda mais sofrimento. A conversa franca, aberta e bem informada entre o médico e o paciente para orientar sobre as possíveis consequências de uma determinada intervenção é uma diretriz presente em vários guidelines de alocação de recursos de UTI, até como forma de sensibilizar os pacientes e familiares de que nem toda obstinação terapêutica vale a pena?.

\footnotetext{
${ }^{7}$ Sobre o tema, o guideline da Suíça dispôs que: "Os pacientes na UTI devem ser avaliados regularmente (pelo menos a cada 48 horas) e interprofissionalmente. Se não houver melhoria, ou houver uma deterioração do estado de saúde, deve ser decidido se o tratamento deve ser continuado ou se o tratamento objetivo deve ser mudado e o paciente deve receber cuidado paliativo. Isso também se aplica a 


\section{Conclusões e Recomendações}

Mesmo antes da pandemia, os profissionais de saúde, aqui incluídos não apenas os "médicos de rotina", mas também os funcionários responsáveis pelas centrais de regulação de leitos, já se confrontavam com situações trágicas para definir quem teria a possibilidade de ocupar ou não os escassos leitos de UTI disponíveis no sistema de saúde. Com a pandemia, houve um rápido incremento da demanda por leitos hospitalares, intensificando a gravidade do problema, inclusive em países que possuem um sistema de saúde mais robusto e bem aparelhado.

Como se viu, em vários lugares do mundo foram elaboradas orientações (guidelines), estabelecendo-se critérios de alocação mais objetivos no intuito de orientar a tomada de decisão em um cenário tão trágico em que a vida ou a morte de seres humanos estão em jogo.

Esses guidelines procuram analisar o problema em seu contexto macro, para evitar um erro muito comum decorrente da formação médica de olhar para o paciente de modo isolado. De um modo geral, são adotados modelos que visam a maximização do bem-estar da coletividade para que os recursos disponíveis possam gerar o maior benefício para a maior quantidade de pessoas. Em algumas situações, isso pode significar a limitação do tratamento para alguns pacientes, o que pode entrar em choque com a deontologia médica, produzindo desconforto, mal-estar e danos psicológicos ao médico, em um contexto em que é imprescindível resguardá-lo de situações difíceis para que ele possa realizar a sua atividade com qualidade.

Além disso, os médicos, pressionados pela necessidade de decidir rapidamente e com um acervo limitado de informações, podem cometer muitos erros morais, ainda que não intencionalmente. Com o aumento da responsabilidade de decidir quem vive e quem morre, os médicos estão mais sujeitos a falhas em suas decisões, seja em razão de seus vieses, seja em razão de uma dificuldade cognitiva causada pelo estresse, pela urgência e pela incerteza. Daí porque os guidelines procuram também minimizar esse desgaste psicológico, oferecendo um parâmetro mais objetivo para retirar o peso decisório dos médicos.

É inquestionável que uma decisão de admitir ou não um paciente em um leito de UTI deve ser baseada em razões essencialmente médicas e, em geral, os guidelines adotam critérios clínicos como parâmetro da alocação de recursos. Porém, em situação de escassez, há outras considerações a serem levadas em conta, como a necessidade de proporcionar a máxima eficácia dos recursos disponíveis sem desrespeitar valores éticos e jurídicos. Por isso, a discussão dos critérios não pode ser puramente médica, devendo levar em conta a contribuição de outras áreas, como a bioética, o direito e a economia.

todos os outros pacientes em tratamento na UTI quando os recursos estão esgotados. A escassez mais aguda de recursos exige mais rigor nos critérios a serem aplicados" (SWISS, 2020, tradução livre). 
Conforme visto, vários países elaboraram parâmetros de priorização de leitos e de ventiladores por conta da COVID-19, levando em conta critérios clínicos mitigados por outros fatores relevantes.

O Brasil ainda não adotou um modelo oficial unificado, embora tenham sido elaboradas propostas por entidades privadas e alguns órgãos públicos em âmbito regional ou estadual. $\mathrm{O}$ ideal é que esses critérios sejam discutidos de modo mais amplo e mais célere, já que não se sabe por quanto tempo a situação dramática provocada pela pandemia pode durar. Afora isso, as situações de escassez podem se dar em ciclos, de modo que há sempre o risco de o problema agravar novamente.

Dentre os protocolos elaborados no Brasil, merece destaque o proposto pela AMIB, não só porque traz critérios objetivos às escolhas, como também porque contempla princípios eticamente relevantes e reconhece a importância de um debate mais amplo para aprimorar o modelo proposto.

A despeito de os custos políticos de estabelecer quais vidas devem ser salvas sejam muito grandes, a discussão precisa ser realizada, pois a total ausência de critérios tem o potencial de trazer resultados muito mais desastrosos, que podem ferir a isonomia consagrada constitucionalmente e dar ensejo à discriminação e a privilégios injustificáveis, além de diminuir a eficácia dos recursos disponíveis.

Em vista disso, a exemplo da Inglaterra, a CONITEC (Comissão Nacional de Incorporação de Tecnologias em Saúde) - órgão análogo ao NICE, poderia estabelecer as regras a serem seguidas, respeitando as peculiaridades e a capacidade de atendimento de cada Estado. O referido órgão está inserido dentro do Ministério da Saúde e conta com a participação do CFM, do Conselho Nacional de Saúde, do Conselho Nacional das Secretarias Estaduais de Saúde (CONASS) e das Secretarias Municipais de Saúde (CONASEMS), da ANS e da ANVISA. Além disso, a CONITEC possui expertise na análise de trade-offs em matéria de saúde, inclusive envolvendo a alocação de recursos escassos, o que a legitima a ser uma potencial reguladora da questão.

Caso se entenda que a CONITEC não tem competência para fixar critérios de prioridade, outros órgãos que integram o Sistema Único de Saúde poderiam fazê-lo, uma vez que possuem legitimidade democrática e condições técnicas para tanto. Em qualquer cenário, nada impede que cada hospital ou sistema local de saúde possa estabelecer critérios de prioridade, de acordo com a disponibilidade de leitos existentes, seguindo a máxima de que quanto maior for a situação de escassez, mais rigorosos devem ser os critérios de admissão e de permanência de pacientes em leitos de UTI. O importante é que os critérios sejam objetivos, transparentes e éticos, observando o objetivo de maximizar o bem-estar da população.

Revista Publicum

Rio de Janeiro, v. 6, n. 1, p. 94-117, 2020

http://www.e-publicacoes.uerj.br/index.php/publicum

DOI: $10.12957 /$ publicum.2020.57573 
Estabelecer critérios, pontuações e limites para a destinação de leitos de UTI e respiradores não é tarefa fácil. As soluções apresentadas podem gerar muitos questionamentos por parte da sociedade, mas há pontos que representam certezas. Não há dúvidas de que fatores como raça, etnia, classe social e religião, assim como outros que não tenham qualquer relação com a possibilidade de recuperação do paciente não podem ser levados em conta. Os demais pontos, que tenham impacto ou relação com a condição clínica do paciente, devem ser submetidos ao debate. Como visto, há muitos direcionamentos que podem ser feitos, já existindo uma boa base de conhecimento produzido nessa área para servir de parâmetro.

Outra questão importante para ser debatida é o momento de extubação do paciente, seja porque ele já não necessita mais da ventilação mecânica, seja porque ele não obteve melhora e deve deixar o aparelho para outro paciente com melhores condições de sobrevivência. Tal decisão é atribuição do médico intensivista, mas os órgãos de triagem podem auxiliá-lo, sobretudo para diminuir os ônus psicológicos derivados de uma decisão tão relevante. Também quanto a isso, é necessário que sejam feitos guidelines orientadores da conduta do médico, pelas mesmas razões expostas acima em relação à alocação de leitos e ventiladores, inclusive estabelecendo o tempo mínimo de observação do quadro clínico do paciente antes que a decisão possa ser tomada.

O intuito da fixação de critérios não é tolher a liberdade do profissional, mas sim dar segurança para que ele possa fazer as escolhas trágicas com maior tranquilidade. Os guidelines mais avançados costumam prever cláusulas de flexibilização para permitir que o médico tenha uma certa discricionariedade para decidir, em contextos reais, o que é melhor a fazer. As escolhas trágicas realizadas pelos médicos em situação de emergência, desde que tomadas de boa-fé, não podem levar à sua responsabilização jurídica, ainda que em retrospectiva possam ser consideradas erradas. Isso vale tanto para o caso de haver critérios mais específicos de alocação de leitos quanto na inexistência deles. Essa é premissa da qual não se pode afastar, sob pena de se atribuir mais um fator de preocupação para os profissionais de saúde, que já estão submetidos a uma elevada carga emocional e cognitiva.

É importante mencionar que os critérios de exclusão/admissão mais restritivos somente devem ser aplicados enquanto durar a situação de escassez. Se o sistema de saúde for capaz de atender a uma gama maior de pacientes, devem ser aplicados os critérios previstos para situação de normalidade. Por isso, os escores de corte não precisam ser fixos, podendo variar de acordo com a necessidade imposta pela demanda e pela capacidade de atendimento, de acordo com as projeções epidemiológicas e modelos estatísticos de evolução da pandemia. O guideline da Suíça, por exemplo, prevê dois protocolos: um para lidar com a escassez quando ainda não há o esgotamento dos leitos e outro para quando ocorrer o colapso. No segundo caso, há uma 
mudança não apenas nos critérios de admissão, mas também nas diretrizes terapêuticas e nos critérios de extubação (SWISS, 2020).

Por fim, em vista da possibilidade de judicialização de pedidos de leitos em UTI, entendemos que o juiz não deveria interferir nas escolhas médicas, seja porque não possui capacidade técnica para saber quem deve ou não receber o tratamento crítico, seja porque não dispõe de todos os elementos necessários para decidir sobre alocação de recursos escassos em saúde, especialmente em pandemia. A intromissão do judiciário em tais casos pode ser desastrosa e deve ser feita apenas em último caso, quando ficar demonstrado ter havido favorecimento indevido de um paciente. Ainda assim, a melhor opção ao juiz é recomendar que a regulação corrija seu erro, de maneira a restabelecer a ordem de prioridade existente. Afinal, mesmo que tenha havido uma má aplicação dos critérios de admissão, o paciente que ingressou com a ação judicial pode não ter preferência em relação a outros pacientes. Assim, o deferimento do seu pedido pode prejudicar ainda mais a ordem existente, em detrimento de outros pacientes que teriam mais prioridade.

\section{Referências}

ARIE, Sophie. Covid-19: Can France's ethical support units help doctors make challenging decisions? BMJ, v. 369, p. m1291, 2020. Disponível online: https://tinyurl.com/y9evp9y4. Acesso em 27/4/2020.

BAKER, Mike \& FINK, Sheri. At the Top of the Covid-19 Curve, How Do Hospitals Decide Who Gets Treatment? New York Times, 31 de março de 2020. Disponível online: https://tinyurl.com/tfyfka4. Acesso em 27/4/2020.

CECHINEL, Clovis. A condição de fragilidade física de idosos e aptidão para a direção veicular. Dissertação de mestrado: UFPR, 2015. Disponível online: https://tinyurl.com/ycy2vta8.Acesso em $27 / 4 / 2020$.

$\mathrm{CHO}$, Hee Cheol. Age is just a number: frailty better evaluates age-dependent heart rhythm defects. The Journal of physiology, v. 594, n. 23, p. 6805, 2016. Disponível online: https://tinyurl.com/ycqf2am6. Acesso em 27/4/2020.

DUBNER, Stephen J. Who Gets the Ventilator? A New Freakonomics Radio Podcast. Ep. 413. Entrevistados: Parag Pathak, Zeke Emanuel e MeiLan Han. Publicado em 15 de abril de 2020. Disponível online: https://tinyurl.com/ydbv72sy. Acesso em 27/4/2020.

EMANUEL, Ezekiel J. et al. Fair allocation of scarce medical resources in the time of Covid-19. 2020. The New England Journal of Medicine, 23 de março de 2020. Disponivel online: https://tinyurl.com/yx5dasqb. Acesso em 27/4/2020. 
FOSTER, Peter. NHS 'score' tool to decide which patients receive critical care. Financial Times, 13 de abril de 2020. Disponível online: https://tinyurl.com/yb3vny2m. Acesso em 27/4/2020.

GOVERNMENT OF CANADA. Clinical management of patients with moderate to severe COVID19 - Interim guidance. Disponível on-line: https://tinyurl.com/y9z5h723. Acesso em 27/4/2020.

KRETZER, Lara e OUTROS. Protocolo AMIB de Alocação de Recursos Em Esgotamento Durante a Pandemia por COVID-19. Associação de Medicina Intensiva Brasileira - AMIB, 24 de abril de 2020. Disponível online: https://tinyurl.com/ydcpk52g. Acesso em 27/4/2020.

KRETZER, Lara e OUTROS. Recomendações da AMIB (Associação de Medicina Intensiva Brasileira), ABRAMEDE (Associação Brasileira de Medicina de Emergência, SBGG (Sociedade Brasileira de Geriatria e Gerontologia) e ANCP (Academia Nacional de Cuidados Paliativos) de alocação de recursos em esgotamento durante a pandemia por COVID-19. Associação de Medicina Intensiva Brasileira - AMIB, 01 de maio de 2020. Disponível online: https://tinyurl.com/yda32rgp. Acesso em 19/5/2020.

MANIR, Mônica. Funil para UTI. Revista Piauí, 22 de abril de 2020. Disponível online: https://piaui.folha.uol.com.br/funil-para-uti/. Acesso em 27/4/2020.

MICHALOWSKI, Sabine et al. Triage in the COVID-19 Pandemic Bioethical and Human Rights Considerations. Technical Report. Essex Autonomy Project and the Ethics of Powerlessness Project, University of Essex, 2020.

MOUNK, Yascha. The extraordinary decisions facing Italian doctors. The Atlantic, 11 de março de 2020. Disponível online: https://tinyurl.com/r5ycs7e. Acesso em 27/4/2020.

MURPHY, Sean. Introduction to Health Utilities and QALYs. Cherish Research, 11 de abril de 2018. Disponível online: https://tinyurl.com/y9m89ldb. Acesso em 27/2/2020.

NICE - National Institute for Health and Care Excellence. COVID-19 rapid guideline: critical care in adults (NG159). Disponível on-line: https://tinyurl.com/y9v3fkjs. Acesso em 27/4/2020.

PATHAK, Parag e outros. Triage Protocol Design for Ventilator Rationing in a Pandemic: Integrating Multiple Ethical Values through Reserves. National Bureau of Economic Research, NBER Working Paper 26951. Publicado em abril de 2020. Disponível online: https://tinyurl.com/y6wn264k. Acesso em 27/4/2020.

SCALPEL, Skeptical. Mortality rate of COVID-19 patients on ventilators. Physician's Weekly. Disponível on-line: https://tinyurl.com/tpuqg9n. Acesso em 27/4/2020.

SOLNICA, Amy; BARSKI, Leonid; JOTKOWITZ, Alan. Allocation of scarce resources during the COVID-19 pandemic: a Jewish ethical perspective. Journal of Medical Ethics, 2020. Diponível online: https://tinyurl.com/y7ggw7tz. Acesso em 27/4/2020.

SWISS, Academy Of Medical Sciences. COVID-19 pandemic: triage for intensive-care treatment under resource scarcity. Swiss medical weekly, v. 150, p. w20229, 2020. Disponível online: https://smw.ch/article/doi/smw.2020.20229. Acesso em 27/4/2020. 
VERGANO, Marco e outros. Clinical Ethics Recommendations for the Allocation of Intensive Care Treatments in exceptional, resource-limited circumstances - Version n. 1. Italian Society of Anesthesia, Analgesia, Resuscitation and Intensive Care (SIAARTI). Disponível on-line: https://tinyurl.com/t5mjsnx. Acesso em 27/4/2020.

WEINSTEIN, Milton C.; TORRANCE, George; MCGUIRE, Alistair. QALYs: the basics. Value in health, v. 12, p. S5-S9, 2009. Disponível online: https://tinyurl.com/yclvsavo. Acesso em 27/4/2020.

WHITE, Douglas B. A Model Hospital Policy for Allocating Scarce Critical Care Resources. University of Pittsburgh School of Medicine, 23 de março de 2020. Disponível online: https://tinyurl.com/y7j93u4l. Acesso em 27/4/2020.

WHITE, Douglas B. et al. Who should receive life support during a public health emergency? Using ethical principles to improve allocation decisions. Annals of Internal Medicine, v. 150, n. 2, p. 132-138, 2009.

WHITE, Douglas B.; LO, Bernard. A framework for rationing ventilators and critical care beds during the COVID-19 pandemic. Jama Network, 27 de março de 2020. Disponível online: https://tinyurl.com/vgfqhnu. Acesso em 27/4/2020.

\section{George Marmelstein}

Professor de Direito Constitucional e Filosofia do Direito na FA7. Doutor em Direito pela

Universidade de Coimbra. Juiz Federal da JFCE.

Ana Carolina Morozowski

Juíza Federal da JFPR.

Enviado em: 04 de fevereiro de 2021

Aprovado em: 04 de fevereiro de 2021 